\title{
EFFECT OF FEATURE SMOOTHING FOR ROBUST SPEECH RECOGNITION
}

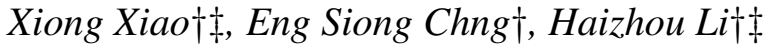 \\ $\dagger$ School of Computer Engineering, Nanyang Technological University, Singapore \\ $\ddagger$ Institute for Infocomm Research, Singapore
}

\begin{abstract}
One class of feature enhancement techniques improve features' robustness by performing temporal filtering to smooth the feature trajectories. While smoothing can enhance the features' robustness by reducing the intra-class variation of the features, it also compromises the features' discriminative power by reducing their inter-class distance. In this paper, we investigate the effect of feature smoothing on speech recognition performance. To evaluate how different degrees of smoothing will affect the performance, the speech features are low-pass filtered with different cut-off frequencies and then used for model training and recognition. From the experimental results, we have two observations: 1) the noisy speech needs more aggressive feature smoothing; 2) the large vocabulary Aurora-4 task prefers less smoothing than the small vocabulary Aurora-2 task.
\end{abstract}

Index Terms - Robust speech recognition, temporal filtering, feature smoothing, modulation frequency, Aurora

\section{INTRODUCTION}

Speech recognition under noisy environment is a challenging problem. The performance of an automatic speech recognition (ASR) system normally degrades substantially in the presence of environmental distortions, such as additive background noises and channel distortions. The performance degradation is mainly due to the statistical mismatch between the clean-trained acoustic model and the noisy test features.

The distortion of spectral features is a clear manifestation of such mismatch. Many efforts have been devoted to reduce the effects of environmental distortions, e.g., the cepstral mean normalization (CMN) [1], the cepstral variance normalization (CVN) [2] and the histogram equalization [3]. These techniques normalize the probability distribution of the features and are therefore called feature normalization techniques.

The temporal filters are another group of techniques to reduce the mismatch by filtering the feature trajectories [4-12]. These temporal filters are design from different perspectives, e.g., the RelAtive SpecTrA (RASTA) filter [4] and the MVA filter [5] are empirically designed to remove the very low and/or high modulation frequencies that are believed to be less relevant to speech intelligibility but prone to environmental distortions. The data-driven filters [6-8] are designed from the data to better represent the features or improve the features' discriminative ability. The temporal structure normalization (TSN) filter [9-11] and the temporal smoothing (TES) filter [12] are designed to normalize the inter-frame correlation of the feature. Although designed from very different criteria, the resulting temporal filters are usually band-pass or low-pass.

The temporal filtering of the features affects the modulation frequency of the features. Two related works attempted to understand the importance of different modulation frequency ranges for human comprehension [13,14] and ASR [15]. Experimental results from both works suggest that the intelligibility of the human speech is mainly related to the $1-16 \mathrm{~Hz}$ modulation frequency range, with the frequencies around $4 \mathrm{~Hz}$ be the most important. In [15], experiments were carried out using different system configurations, which include the type of noise corruption, the signal-to-noise ratio (SNR), the type of feature, the type of recognizer and the database used for evaluation. Results show that for different configurations, the useful modulation frequency range of the feature for speech recognition are usually from $1-16 \mathrm{~Hz}$.

In this paper, we extend on [15] to examine how environmental condition and vocabulary size of recognition task will affect the useful modulation frequency range. In particular, we examine the effect of removing high modulation frequency, i.e. feature smoothing, for various SNR levels and two tasks with different vocabulary sizes. The feature smoothing can have a mix of positive and negative effects on acoustic modeling. On one hand, smoothing improves the features' robustness by reducing the features' variations in different environmental conditions. On the other hand, smoothing may compromise the features' discriminative power between acoustic models. Hence, the final recognition performance is a trade-off between the two effects, which are both affected by the cut-off frequency of the low-pass temporal filter.

This paper is organized as follows. In section 2 we describe the normalization technique and low-pass filters used in our experiments. In section 3, the experimental results and discussions are presented. Finally, we conclude in section 4.

\section{FEATURE PROCESSING}

We propose a feature smoothing process as shown in Fig. 1. Every raw speech feature trajectory undergoes a two-stage processing to produce the final features for recognition. Each dimension of speech features is processed independently. The processing stages include the mean and variance normalization (MVN) and the feature smoothing. In this section, we describe these two stages individually.

\subsection{Feature Normalization}

Noise corruption changes the probability distribution of speech features significantly, e.g., the mean and variance. If the noisy features have very different means and variances from the clean features, the effect of feature smoothing is less observable especially at low SNR level. To study the effect of feature smoothing more effectively, the MVN is applied to all the features prior to the filtering. The use of MVN as a preprocessing unit is a common practice for several temporal filters, such as the MVA [5], the TSN [9] and the TES [12]. 


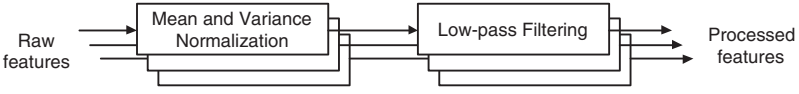

Fig. 1. The two-stage feature processing.

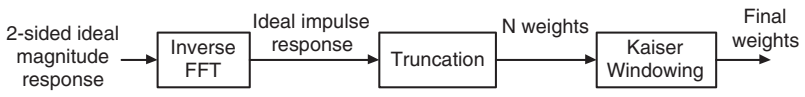

Fig. 2. The procedures of designing the low-pass filters.

\subsection{Feature Smoothing}

Noncausal finite impulse response (FIR) low-pass filters are used to smooth the MVN-normalized features. The filters have odd number (N) of taps and the weights are symmetrical w.r.t. the central tap. Due to the filters' transient periods at both ends of an utterance, the $(\mathrm{N}-1) / 2$ frames at the two ends of every utterance cannot be filtered and their smoothness may be very different from that of the filtered frames. To reduce this inconsistency, the unfiltered frames are removed after the filtering. To retain more frames, the filters' length should be as short as possible and yet provide acceptable magnitude response. In our experiments, we choose $\mathrm{N}=11$ taps, and hence the first and last 5 frames of every utterance are removed after filtering.

The FIR low-pass filters are designed using the Window-based method [16] (See Fig. 2). The two-sided ideal magnitude response of the low-pass filter (the step response) is sampled in the frequency domain. The ideal impulse response of the filter is then obtained by applying the inverse fast Fourier transform (IFFT) to the samples of the magnitude response. Only the central $\mathrm{N}$ weights are used and the Kaiser window is multiplied to the $\mathrm{N}$ weights to reduce the truncation effect. The beta of the Kaiser window is used to control the characteristics of the final filter response. Generally, a large beta can reduce the level of ripples in the passband and stopband but also results in slower transition from passband to stopband. In our experiments, large beta values are used for high cut-off frequency filters to reduce the ripples and small beta values are used for low cut-off frequency filters to achieve fast transition.

As the purpose here is to study the effect of feature smoothing rather than accurately measure the importance of the modulation frequencies, we only empirically choose eight filters in the experiments. The cut-off frequencies of the filters and the corresponding beta values are listed in Table 1 and the magnitude responses of the filters are shown in Fig. 3.

\section{EXPERIMENTAL SETUP AND RESULTS}

\subsection{Feature Extraction Settings}

The $39 \mathrm{Mel}$ frequency cepstral coefficients (MFCC) $(13$ static +13 delta +13 acceleration) are used as the features for speech recognition. The first MFCC coefficient c0 (i.e. the cepstral energy) is used instead of the log energy as the MVN works better with the c0 [5]. After the 39 raw features are extracted, the MVN and the low-pass filters are applied on each feature trajectory in sequence. Both the training data and the test data are processed identically. The frame length is $25 \mathrm{~ms}$ and the frame shift is $10 \mathrm{~ms}$. Hence the frame rate is $100 \mathrm{~Hz}$ and the maximum modulation frequency is $50 \mathrm{~Hz}$.
Table 1. List of cut-off frequencies $\left(f_{c}\right)$ and the corresponding beta values of the Kaiser window used in the filter design.

\begin{tabular}{|c|c|c|c|c|c|c|c|c|}
\hline$f_{c}(\mathrm{~Hz})$ & 6 & 8 & 10 & 12 & 15 & 20 & 30 & 40 \\
\hline beta & 0.5 & 0.5 & 1 & 2 & 3 & 4 & 4 & 4 \\
\hline
\end{tabular}

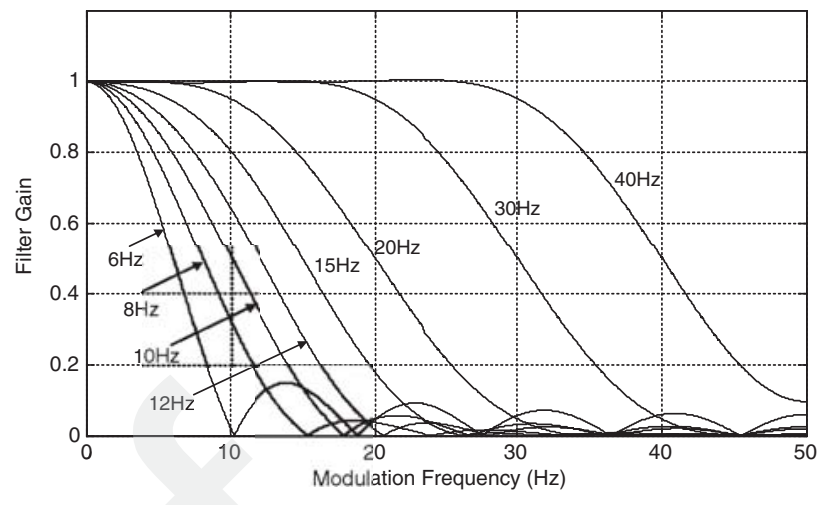

Fig. 3. The filters' magnitude responses with different cut-off frequencies.

\subsection{Evaluation Measure}

We study the effect of feature smoothing by comparing its effect in recognition accuracy. Specifically, we compare how feature smoothing with different cut-off frequencies improves or degrades the performance w.r.t. the MVN baseline. To make the results of different SNR levels comparable, we use the relative error rate reduction (RER) as the measure. The RER is calculated by the equation: $\operatorname{RER}=\left(E_{b}-E_{s}\right) / E_{b} \times 100 \%$, where $E_{b}$ and $E_{s}$ are the recognition error rate obtained from the MVN baseline and the MVN+smoothing, respectively.

\subsection{Experiments on Aurora-2 Task}

The clean-condition training scheme defined in the Aurora- 2 task is used. The settings of the recognizer follow the standard Aurora-2 procedure [17]. The test data of the Aurora- 2 task are divided into 10 environmental distortion cases, with each case being divided into seven SNR levels: clean plus decreasing SNR levels from 20dB to $-5 \mathrm{~dB}$ with $5 \mathrm{~dB}$ step. The first eight distortions are additive noises. These eight noises are recorded in typical real world environments, such as in a subway train. The noises are added to the clean speech in different SNR levels artificially. The last two distortions are mix of channel filtering and additive noise.

The MVN baseline results on Aurora- 2 task are shown in Table 2, and the results are comparable to the state-of-the-art performance for MVN [5]. The RER curves for different SNR levels are shown in Fig. 4. The ASR performance is improved over the baseline if RER is greater than zero. It is observed that the trends of the RER curves are different for different SNR levels. For the clean case, the RER drops almost consistently as the cut-off frequency $\left(f_{c}\right)$ decreases. For noisy cases, as the $f_{c}$ decreases, the RER first increases and then decreases. Furthermore, the noisier cases usually reach the maximum RER at lower $f_{c}$. For example, the $20 \mathrm{~dB}$ and $15 \mathrm{~dB}$ cases attain the maximum RER at $15 \mathrm{~Hz}$, the $10-0 \mathrm{~dB}$ cases' maximum RERs are at $10 \mathrm{~Hz}$ and for the $-5 \mathrm{~dB}$ case, RER continues to increase slowly even at $6 \mathrm{~Hz}$. The maximum RER attainable is 
Table 2. The MVN baseline performance averaged over 10 environmental distortion cases for each SNR level on the Aurora-2 task.

\begin{tabular}{|c|c|l|l|l|l|l|l|}
\hline SNR(dB) & Clean & 20 & 15 & 10 & 5 & 0 & -5 \\
\hline Accuracy(\%) & 99.3 & 97.6 & 95.4 & 89.3 & 74.0 & 43.7 & 16.9 \\
\hline
\end{tabular}

Table 3. The MVN baseline performance averaged for the 4 environmental distortion categories on the Aurora-4 task.

\begin{tabular}{|c|c|c|c|c|}
\hline Category & Clean & Mic & Additive & Additive+Mic \\
\hline Accuracy(\%) & 86.96 & 77.30 & 63.20 & 52.65 \\
\hline
\end{tabular}

also inversely proportional to the SNR level loosely. In addition, the RER of the cleaner cases generally degrades faster than that of the noisier cases. For example, after both reaching the maximum RER at $15 \mathrm{~Hz}$, the $20 \mathrm{~dB}$ RER drops faster than the $15 \mathrm{~dB}$ RER. For most of the SNR levels, the performance drops significantly when the $f_{c}$ is below $12 \mathrm{~Hz}$. This agrees with the observations in [15].

The results in Fig. 4 show that the optimal cut-off frequency is related to the SNR of the signal. Generally, high SNR cases prefer less smoothing than low SNR cases for the Aurora-2 task.

\subsection{Experiments on Aurora-4 Task}

To evaluate whether the RER performance is affected by the number of acoustic classes in the recognizer, the large vocabulary Aurora-4 task is also used for evaluation. The recognizer's settings are similar to the reference system described in [18] (see [11] for more details about our system settings). There are 14 test cases in the Aurora-4 task and for each case, 330 test utterances are used. The test cases are grouped into 4 categories, i.e. the clean category (case 1), the additive noise category (case 2-7, with noise distortion similar to that of Aurora-2), the microphone mismatch category (case 8) and the additive noise plus microphone mismatch category (case 9-14). We rank the four categories according to their degree of environmental distortion: clean $<$ microphone mistmatch $<$ additive noise $<$ additive noise +microphone mismatch.

The MVN baseline results on Aurora- 4 task shown in Table 3 are comparable to the published results [11]. The RER curves of the four categories are shown in Fig. 5. From the figure, the RER curves show a similar pattern as that in Fig. 4, i.e. the RER drops faster for categories with lower degree of environmental distortion as the cut-off frequency decreases. This further shows the dependency of the optimal cut-off frequency on the environment condition. We note that the RER improvement on the Aurora- 4 task is generally much less significant than that on the Aurora-2 task. We compare the performance on the two tasks in details in the next subsection.

\subsection{Comparison of Aurora-2 and Aurora-4 Tasks}

To compare the two tasks, the clean and additive noise categories of both tasks are used. For Aurora-2, it is necessary to create an additive noise category comparable to that of the Aurora- 4 . This is achieved by matching the type of noise distortions and SNR levels of the Aurora-2 to their Aurora-4 counterpart. Specifically, six noise types: car, babble, restaurant, street, airport and train station; and three SNR levels: $15 \mathrm{~dB}, 10 \mathrm{~dB}$ and $5 \mathrm{~dB}$ from the Aurora-2 task are used to form the additive noise category.

The RER curves of the two tasks are shown in Fig. 6. From the figure, the clean category RER curves of the two tasks show similar trend, i.e. the smoothing will reduce the RER performance. However, for additive noise category, the RER curves are quite different:

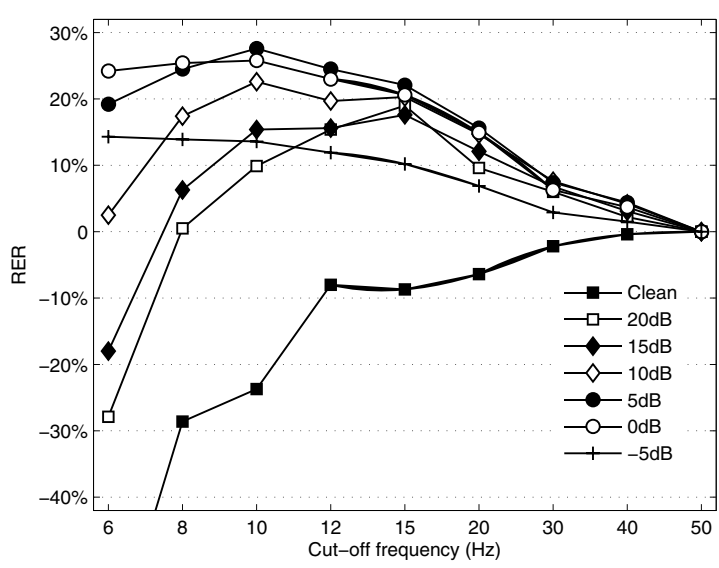

Fig. 4. The effect of feature smoothing on the Aurora-2 task with different cut-off frequencies. The results are obtained by averaging over the 10 noise cases for each SNR level. The $50 \mathrm{~Hz}$ case is the MVN baseline without feature smoothing.

1) the Aurora- 2 reaches maximum RER at $10 \mathrm{~Hz}$ and for Aurora- 4 it is $20 \mathrm{~Hz}$. 2) the improvement on Aurora- 2 is much larger than that of the Aurora-4. The results show that the feature smoothing has different effects on tasks with different vocabulary sizes.

\subsection{Discussion}

Although the speech intelligibility was found to be mainly related to the low modulation frequency range $(<16 \mathrm{~Hz})[13-15]$, we find that the contribution of the frequencies to speech recognition is related to the environmental condition and task vocabulary size. From the viewpoint of pattern classification, the discriminative power and the robustness of the speech feature can be contradictory requirements. In the case of noisy speech recognition, we assume that the noise distortion exist in all modulation frequencies. By using a lower cut-off frequency, more distortion can be removed. However, filtering with low cut-off frequency will remove speech information and hence reduce the features' discriminative power significantly. The optimal cut-off frequency should be chosen to balance the two effects. Experimental results show that the optimal cut-off frequency is related to both the environmental condition and the task vocabulary. Generally, cleaner features prefer to be less smoothed to retain more speech information, and noisier features can be smoothed more deeply to remove more distortion. In addition, small vocabulary Aurora-2 task can be smoothed more severely than the large vocabulary Aurora-4 task. This is because for small vocabulary tasks, the models of different sound classes are well separated and therefore the system can tolerate greater loss of discriminative power in exchange for higher degree of robustness. The study shows that a fixed temporal filter cannot be optimal for various environmental conditions and vocabulary sizes. It may be good for the temporal filters to adapt their cut-off frequency to these factors.

\section{CONCLUSIONS}

In this paper, we studied the effect of feature smoothing for speech recognition. Experimental results show that feature smoothing affects the recognition performance differently in various SNR lev- 


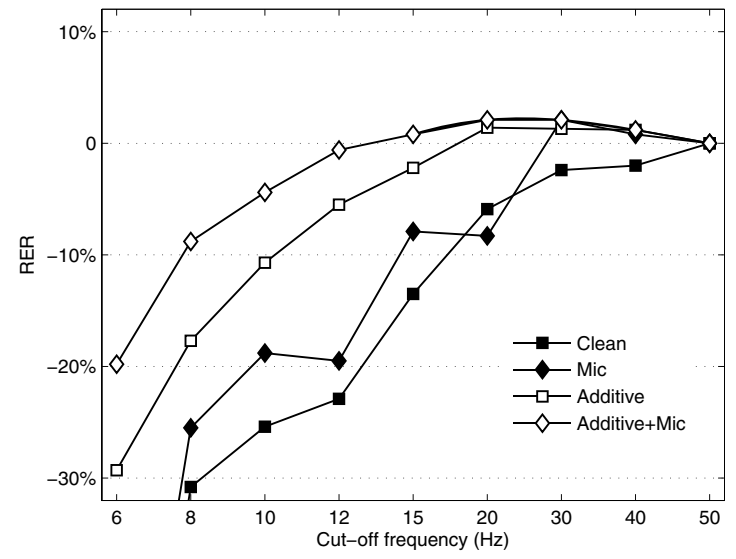

Fig. 5. The effect of feature smoothing on the Aurora- 4 task with different cut-off frequencies. The results are obtained by averaging over the noise cases in each category.

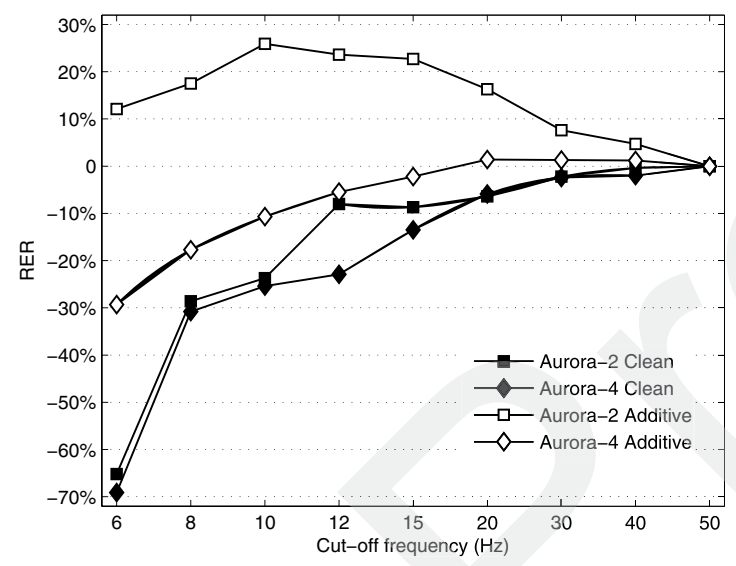

Fig. 6. Comparison of the performance on the Aurora-2 and Aurora4 tasks.

els and vocabulary sizes. The recognition performance is jointly affected by features' robustness and discriminative power, which are both controlled by the cut-off frequency of the smoothing filter.

\section{REFERENCES}

[1] S. Furui, "Cepstral analysis technique for automatic speaker verification," IEEE Trans. Acoustics, Speech and Signal Processing, vol. 29, no. 2, pp. 254-272, 1981.

[2] Olli Viikki and Kari Laurila, "Cepstral domain segmental feature vector normalization for noise robust speech recognition," Speech Communication, vol. 25, pp. 133-147, 1998.

[3] A. de la Torre, A. M. Peinado, J. C. Segura, J. L. PerezCordoba, M. C. Benitez, and A. J. Rubio, "Histogram equalization of speech representation for robust speech recognition," IEEE Trans. Speech and Audio Processing, vol. 13, no. 3, pp. 355-366, 2005.
[4] H. Hermansky and N. Morgan, "RASTA processing of speech," IEEE Trans. Speech and Audio Processing, vol. 2, no. 4, pp. 578-589, 1994.

[5] C.-P. Chen and J. A. Bilmes, "MVA processing of speech features," IEEE Trans. Audio, Speech, and Language Processing, vol. 15, no. 1, pp. 257-270, 2007.

[6] S. van Vuuren and H. Hermansky, "Data-driven design of RASTA-like filters," in Proc. Eurospeech '97, Rhodes, Greece, Sept. 1997.

[7] M. L. Shire, "Data-driven modulation filter design under adverse acoustic conditions and using phonetic and syllabic units," in Proc. Eurospeech '99, Budapest, Hungary, Sept. 1999.

[8] J.-W. Hung and L.-S. Lee, "Optimization of temporal filters for constructing robust features in speech recognition," IEEE Trans. Audio, Speech, and Language Processing, vol. 14, no. 3, pp. 808-832, 2006.

[9] Xiong Xiao, Eng Siong Chng, and Haizhou Li, “Temporal structure normalization of speech feature for robust speech recognition," IEEE Signal Processing letters, vol. 14, no. 7, pp. 500-503, 2007.

[10] Xiong Xiao, Eng Siong Chng, and Haizhou Li, "Normalizing the speech modulation spectrum for robust speech recognition," in Proc. ICASSP '07, Honolulu, USA, May 2007, pp. 1021-1024.

[11] Xiong Xiao, Eng Siong Chng, and Haizhou Li, "Evaluating the temporal structure normalisation technique on the Aurora4 task," in Proc. Eurospeech '07, Antwerp, Belgium, Sept. 2007, pp. 1070-1073.

[12] L. García, J.C. Segura, C. Benítez, J. Ramírez, and A. de la Torre, "Normalization of the inter-frame information using smoothing filtering," in Proc. INTERSPEECH '06, Pittsburgh, USA, Sept. 2006, pp. 369-372.

[13] Rob Drullman, Joost M. Festen, and Reinier Plomp, "Effect of temporal envelope smearing on speech reception," Journal of the Acoustical Society of America, vol. 95, no. 2, pp. 10531064, 1994.

[14] Rob Drullman, Joost M. Festen, and Reinier Plomp, "Effect of reducing slow temporal modulations on speech reception," Journal of the Acoustical Society of America, vol. 95, no. 5, pp. 2670-2680, 1994.

[15] N. Kanedera, T. Arai, H. Hermansky, and M. Pavel, "On the relative importance of various components of the modulation spectrum for automatic speech recognition," Speech Coтmunication, vol. 28, no. 1, pp. 43-55, 1999.

[16] J. G. Proakis and D. G. Manolakis, Digital signal processingprinciples, algorithms, and applications, Prentice-Hall, 3 edition, 1996.

[17] D. Pearce and H.-G. Hirsch, "The AURORA experimental framework for the performance evaluation of speech recogntion systems under noisy conditions," in Proc. ICSLP '00, Beijing, China, Oct. 2000, vol. 4, pp. 29-32.

[18] N. Parihar and J. Picone, "Aurora working group: DSR front end LVCSR evaluation AU/384/02," Tech. Rep., Institute for Signal and Infomation Processing, Mississippi State Univ., MS, Dec. 2002 